\title{
Surgical treatment strategy for locally advanced colorectal cancer with abdominal wall invasion
}

\author{
Zhicheng Song ${ }^{1,2 \#}$, Dongchao Yang ${ }^{1,2 \#}$, Heng Song ${ }^{2}$, Wenpei Dong ${ }^{2}$, Jugang $\mathbf{W u}^{2}$, Jianjun Yang ${ }^{2}$, Yan Gu ${ }^{1,2}$ \\ ${ }^{1}$ Huadong Hospital Affiliated to Fudan University, Shanghai, China; ${ }^{2}$ Shanghai Ninth People's Hospital, Shanghai Jiao Tong University School of \\ Medicine, Shanghai, China \\ Contributions: (I) Conception and design: Z Song, Y Gu; (II) Administrative support: Y Gu; (III) Provision of study materials or patients: Z Song, \\ D Yang; (IV) Collection and assembly of data: J Wu, W Dong; (V) Data analysis and interpretation: H Song, J Yang; (VI) Manuscript writing: All \\ authors; (VII) Final approval of manuscript: All authors. \\ \#These authors contributed equally to this work. \\ Correspondence to: Yan Gu, MD, PhD. Huadong Hospital Affiliated to Fudan University, 221 West Yan'an Road, Shanghai, China. \\ Email: yangu@shsmu.edu.cn.
}

\begin{abstract}
Background: The incidence of abdominal wall metastasis from colorectal cancer (CRC) is very low, but it has a poor prognosis. Despite the advances in radiotherapy, chemotherapy, and targeted therapy, patient prognosis has not improved significantly. Through surgical treatment, some patients with locally advanced CRC with abdominal wall invasion can achieve tumor-free survival or an improved quality of life.

Methods: The clinical data of 15 patients in our department from January 2015 to January 2020 were retrospectively analyzed. All patients underwent preoperative three-dimensional reconstruction of the tumor and abdominal wall after discussion with a multidisciplinary team (MDT). Patient information, including tumor size, defect size, operation time, intraoperative bleeding, hospital stay, and other factors, was collected. Results: All 15 patients underwent resection followed by reconstruction for locally advanced CRC with abdominal wall invasion. The average tumor area and abdominal wall defects were $98.13 \pm 71.70$ and $270.07 \pm 101.95 \mathrm{~cm}^{2}$, respectively; and accurate abdominal wall classification and zoning were obtained for all patients. The average operation time was $431.7 \pm 189.2 \mathrm{~min}$, and the average blood loss was $513.3 \pm 244.6 \mathrm{~mL}$. The recurrence rates in the incisional hernia and abdominal wall were $6.0 \%$ and $13.3 \%$, respectively. The patient survival rate was $87.7 \%$.
\end{abstract}

Conclusions: Surgical treatment of locally advanced CRC with abdominal wall invasion is feasible, but requires accurate and comprehensive preoperative evaluation.

Keywords: Three dimensional visualization; complex abdominal wall defects; locally advanced colorectal cancer with abdominal wall invasion (locally advanced CRC with abdominal wall invasion); abdominal wall reconstruction

Submitted Mar 22, 2021. Accepted for publication May 17, 2021.

doi: 10.21037/atm-21-2094

View this article at: http://dx.doi.org/10.21037/atm-21-2094

\section{Introduction}

Colorectal cancer (CRC) is the third most common tumor in men and the second most common tumor in women, accounting for $10 \%$ of all tumor types worldwide. With more than 600,000 deaths estimated each year, CRC is the fourth leading cause of cancer-related death globally $(1,2)$. According to the China Cancer Report in 2018, CRC ranked third and fifth among all malignant tumors in terms of incidence and mortality in China, with 376,000 new cases and 191,000 deaths, respectively (3).

It is common for CRC patients to have distant metastases. Among them, $40-50 \%$ of patients have liver metastases, $10-15 \%$ have lung metastases, and $4-19 \%$ have peritoneal metastases, while metastases to the bone, brain, and other locations, such as the adrenal glands and spleen, are relatively rare $(4,5)$. Peritoneal metastasis of CRC is the third most common site after liver and lung metastases, 
and $5-15 \%$ of CRC patients have a peritoneal metastasis at the time of diagnosis. The median survival of patients with CRC and peritoneal metastasis after systemic chemotherapy alone is only 5.2-7.0 months, while the median survival of patients with malignant intestinal obstruction is 3.0-3.5 months (6). The 5-year survival rate of patients with CRC combined with peritoneal metastasis is only $20-25 \%$, and the median survival time of these patients is only 6-9 months after being diagnosed. The 1-year survival rate of patients with malignant ascites is $<10 \%$ (7).

However, the incidence of abdominal wall invasion by a metastasis after colon cancer surgery is very low. Reilly et al. studied 1,711 patients with colon cancer and found that the incidence of postoperative incision recurrence was $0.64 \%$; the average follow-up was 1.8 years, and 3 of 11 patients were still alive with high mortality rate (8). In recent years, the effectiveness of CRC chemotherapy, targeted therapy, and immunotherapy has gradually improved. Despite improvements, the overall treatment effect is not ideal. Neoadjuvant chemoradiotherapy not only can reduce tumor size and recurrence, but also increase the tumor resection rate and anus retention rate with very slight side effect (9). National Comprehensive Cancer Network (NCCN) guidelines recommend preoperative concurrent chemoradiotherapy as a priority standard treatment for II/III rectal cancer. With improved surgical techniques and the addition of neoadjuvant radiation therapy, 5-year local recurrence rates have decreased from $>25 \%$ to approximately $5 \%$ to $10 \%$ (9). However, distant metastatic disease remains the most significant cause of death for these patients.

In this study, we conducted a retrospective analysis and discussion of 15 cases of CRC with local invasion of the abdominal wall to explore the feasibility of surgical treatment for such patients.

We present the following article in accordance with the STROBE reporting checklist (available at http://dx.doi. org/10.21037/atm-21-2094).

\section{Methods}

\section{Patients}

We selected 15 colorectal tumors patients with abdominal wall invasion from the General Surgery Department of Shanghai Ninth People's Hospital from January 2015 to January 2020 for inclusion in this retrospective study. This study was approved by the ethics committee of the
Ninth People's Hospital Affiliated to Shanghai Jiaotong University (approval number: 2016-128-177). This study was performed in accordance with the ethical standards of our institutional research committee and the principles of the Declaration of Helsinki (as revised in 2013).

The inclusion criteria were as follows: (I) patients aged 18-85 years old; (II) patients who consented to radical or palliative surgery; (III) the postoperative pathological result was CRC; and (IV) patients signed the informed consent form. The exclusion criteria were as follows: (I) patients with severe organ dysfunction; (II) patients with an inability to tolerate anesthesia; and (III) patients or their family members were unwilling to accept the surgery.

\section{Treatment}

All patients underwent enhanced computed tomography (CT) or positron emission tomography and computed tomography (PET-CT) before surgery to exclude the presence or absence of a distant metastasis of the tumor. Furthermore, all patients received an accurate preoperative assessment, including three-dimensional reconstruction of the tumor and abdominal wall after discussion with a multidisciplinary team (MDT). Information about the patients' tumor size, defect size, operation time, intraoperative bleeding, hospital stay, and other relevant factors was collected.

Image data was imported into Medraw software (Image Medraw Technology Co., Ltd., China) in digital imaging and communications in medicine (DICOM) format for three-dimensional reconstruction. The imaging physician and clinician jointly built a three-dimensional model, which included bone, muscle, blood vessels, urinary system, and abdominal wall tumor lesions, and provided the corresponding records and statistics. Combination, disassembly, rotation, and other methods were used to show the anatomical relationship between the abdominal wall tumors and surrounding important organs, and provide theoretical support for the preparation of individualized surgical plans. Combined with the classification and division of the abdominal wall to determine the type of abdominal wall defect, the clinician can develop an individualized surgery plan to simulate the following: the abdominal wall defect area after abdominal tumor resection (according to the anatomical relationship between the abdominal wall tumor and adjacent tissues and organs), the incision situation, and the presence or absence of tumor metastasis. 
Table 1 Patient characteristics

\begin{tabular}{lc}
\hline Variable & Value or $\mathrm{M} \pm \mathrm{SD}$ \\
\hline Age & $54.9 \pm 12.4$ \\
Gender & 13 \\
Male & 2 \\
Female & \\
With other illnesses & 2 \\
Coronary heart disease & 1 \\
Tuberculosis & 1 \\
Hepatitis B & 1 \\
Hydronephrosis & \\
Tumor area infection & \\
Origin & 11 \\
Colon & \\
Rectum & \\
\hline
\end{tabular}

\section{Follow-up}

Follow-up data were retrospectively obtained from the medical records. Each patient was followed-up every six months, and the final follow-up was on August 16, 2020. Follow-up data included death, flap necrosis, incision infection, mesh infection, intestinal fistula, and other conditions.

\section{Statistical analysis}

All statistical analyses were performed using IBM SPSS Statistics 22.0 (IBM SPSS Statistics, IBM Corporation, Armonk, NY, USA). Categorical values were reported as the frequency and percentage, and continuous values were reported as the mean \pm standard deviation $(\mathrm{SD})$ or the median with range, depending on whether the values were normally distributed or not. Categorical variables were statistically analyzed by the chi-square test, and continuous variables were compared using Student's $t$-test or the MannWhitney U test.

\section{Results}

This study included 15 patients (13 males and 2 females). The mean age of the patients was $54.87 \pm 12.39$ years, the mean average hospital stay was $35.23 \pm 11.92$ days, and the
Table 2 Surgical and follow-up data

\begin{tabular}{lc}
\hline Variable & Value or $\mathrm{M} \pm \mathrm{SD}$ \\
\hline Type of defect & 0 \\
Type I & 3 \\
Type II & 12 \\
Type III & $98.13 \pm 71.70$ \\
Tumor area $\left(\mathrm{cm}^{2}\right)$ & $270.07 \pm 101.95$ \\
Abdominal wall defects $\left(\mathrm{cm}^{2}\right)$ & \\
Time of the operation & $431.7 \pm 189.2$ \\
Total & $733.3 \pm 130.2$ \\
Flap & $356.2 \pm 109.9$ \\
No-Flap & \\
Blood & $513.3 \pm 244.6$ \\
Total & $866.7 \pm 94.3$ \\
Flap & $425.0 \pm 183.1$ \\
No-Flap & \\
Complication & 1 \\
Infection of incision & 5 \\
Infection of mesh & \\
Subcutaneous seroma & \\
Hematoma & \\
Abdominal hernia & \\
Fistula & \\
\hline Death & \\
\hline
\end{tabular}

mean American Society of Anesthesiologists (ASA) score was $1.87 \pm 0.52$. Other relevant information is listed in Table 1.

All 15 patients underwent abdominal wall tumor resection. The average tumor area and abdominal wall defects were $90 \mathrm{~cm}^{2}$ and $270 \mathrm{~cm}^{2}$, respectively. The defects were accurately typed and partitioned (Table 2), and all of the cases were diagnosed with adenocarcinoma by postoperative pathology. Three patients underwent flap transplantation, while two patients received vacuum sealing drainage (VSD) to temporarily close the abdominal cavity and then underwent a two-staged abdominal wall reconstruction. The average operation time was $431.7 \pm 189.2 \mathrm{~min}$, and the average blood loss was $50 \mathrm{~mL}$. The average operation time and blood loss for flap transplantation were $70 \mathrm{~min}$ and $80 \mathrm{~mL}$, respectively. The average operation time and blood 

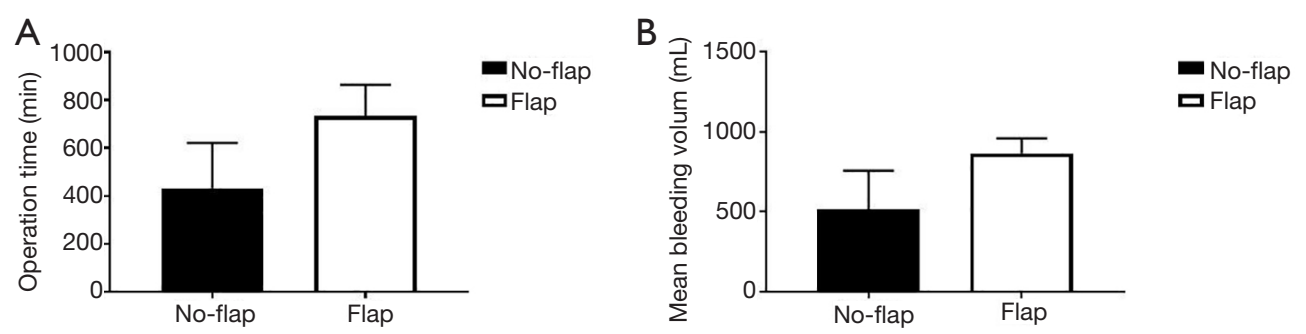

Figure 1 Effect of flap transplantation on operation time and intraoperative blood loss. (A) Mean operation time with or without flap transplantation. (B) Mean operation time and bleeding volume with or without flap transplantation.

loss without flap transplantation were $30 \mathrm{~min}$ and $40 \mathrm{~mL}$, respectively (Figure 1).

The longest follow-up time among the 15 patients was 5 years, and the shortest follow-up time was 4 months. Incision infections occurred in five patients after surgery, and two patients died due to an advanced tumor. One patient developed an abdominal wall hernia, one patient developed a subcutaneous hematoma, and one patient developed an intestinal fistula. The recurrence rates of incisional hernia and abdominal wall tumor were $6.0 \%$ and $13.3 \%$, respectively. The patient survival rate was $87.7 \%$.

\section{Discussion}

Abdominal wall metastasis of CRC is very rare; however, its occurrence signified that the tumor is advanced with a poor prognosis (8). Aggressive surgical treatment will result in a substantial abdominal wall defect, and palliative surgical resection will result in postoperative recurrence, creating a considerable challenge for surgeons. Therefore, conservative treatment often becomes the first choice for such patients. First-line drugs, such as oxaliplatin and irinotecan, for the treatment of patients with CRC have achieved good results. With the progression of targeted therapy and immunotherapy, patients with advanced CRC can achieve long-term survival. However, the overall treatment effect is not ideal. At present, there are still no relevant guidelines or expert consensus regarding the best approach for CRC metastases to the abdominal wall.

Radiotherapy, chemotherapy and neoadjuvant therapy have greatly improved the recurrence and mortality of colorectal cancer after surgery. However, for patients with locally advanced rectal cancer in the abdominal wall, radiotherapy, chemotherapy and neoadjuvant therapy can only achieve a certain period of time to shrink the tumor and prolong the patient's mid-term survival time (10). If the patient is unable to undergo surgical treatment, or the quality of life can be improved only through the above methods; if the patient uses neoadjuvant chemotherapy or radiotherapy to create conditions for surgery, combined with radiotherapy and chemotherapy after surgical treatment can make the patient survive with radical cure or tumorfree survival, prolong the survival period and Improve the quality of life of patients (11). However, patients with locally advanced colorectal cancer often have other organ invasions, multiple organ dysfunctions, etc. Various reasons may increase the risk of surgery and anesthesia, and increase the risk of postoperative death. Accurately assessing the overall and local conditions of the patient before surgery is still the biggest challenge for surgical treatment.

The traditional view is that the treatment of CRC metastases to the abdominal wall should first consider the condition of the primary tumor. If the CRC cannot be controlled or there are other distant metastases in other organs, then the main treatment should be a symptomatic or systemic treatment, such as chemotherapy and radiotherapy. Surgery for CRC metastases to the abdominal wall has high technical requirements because it is difficult to reconstruct the abdominal wall, and the overall prognosis is poor. With the development of modern medical concepts and technologies, as well as the routine use of a comprehensive preoperative evaluation by a MDT, the treatment concept of CRC metastases to the abdominal wall has changed, and active surgical treatment has become a feasible choice $(12,13)$. Through surgery, some patients with locally advanced CRC with abdominal wall invasion can achieve tumor-free survival or an improved quality of life. Patients are often able to tolerate surgery, the tumor can be completely removed, and multiple organ dysfunction or tumor invasion of large blood vessels can increase the risk of perioperative anesthesia and mortality. The treatment strategy is to formulate a personalized treatment plan through accurate assessment of the systemic conditions and MDT discussion (14). If the tumor can be completely 



Figure 2 Surgical procedure for abdominal wall tumor. (A) Preoperative measurement; (B) colorectal cancer with abdominal wall invasion; (C) abdominal wall defect; (D) resection of bowel and abdominal wall tumor; (E) component separation technique; (F) Reconstruction the peritoneum; $(\mathrm{G})$ reconstruction the abdominal wall; $(\mathrm{H})$ setting of the drainage tube.

resection, the combined treatment method such as enlarged resection of the abdominal wall tumor + resection of multiple involved organs combined with postoperative chemotherapy is performed. If the tumor cannot be completely removed, neoadjuvant chemoradiation is the first choice to see whether it can create conditions for surgery. If after treatment, the conditions for surgery are met and surgical treatment is possible. If there is no surgery conditions, local or systemic drug treatment is given (15).

There are two basic categories of surgery, radical resection and palliative resection. Radical resection is based on both radical resection of the colorectal tumor and extended resection of the abdominal wall tumor. In principle, the resection range should extend $2-3 \mathrm{~cm}$ into the normal tissue at the tumor edge, and a rapid intraoperative pathological examination is performed to ensure that there is a clean margin, that no tumor remains within the basal tissues, and that an R0 resection has been achieved. Palliative resection is mainly applied to address the complications of abdominal wall tumors, such as ulceration, bleeding, and infection. The purpose of palliative resection is to enhance the quality of life of the patients and improve their condition so that they may receive additional treatment (Figure 2).

Surgical treatment is feasible for locally advanced CRC with abdominal wall invasion. There are numerous tools available to ensure that the surgery is performed successfully and efficiently. Firstly, the concept of a MDT has been widely used and promoted for tumor treatment. The promotion and application of new technologies, including imaging, pathology, endoscopy, radiotherapy, and chemotherapy, have significantly improved the R0 resection rate of CRC tumors. The MDT combines the advantages of multiple disciplines to accurately assess the patient's condition and formulate a personalized and comprehensive treatment plan (16). Furthermore, it can also more effectively achieve multidisciplinary crossover, perform indepth analyses of problems in each professional aspect, and make comprehensive considerations of the patient's general situation, various organ functions, anesthesia risks, surgical risks, etc. Locally advanced CRC with abdominal wall invasion is different from widespread metastasis. Radical surgery combined with radiotherapy, chemotherapy, and targeted therapy can significantly improve the prognosis of patients.

Surgical resection remains the main potentially curative treatment among patients with resectable liver, lung, and other metastatic tumor $(17,18)$. Accurate preoperative assessment is important before the surgery, including assessment of general situation organ situation which is invaded. The patient's various organs function well, is a prerequisite for surgery and anesthesia. Systemic therapies, newer biologic agents (for example, bevacizumab and cetuximab) and immunotherapeutic agents have revolutionized the treatment options for metastases patients, who are intolerance to surgery (17). The patients 
who are pT3 or pT4, if the metastatic tumor can be resection R0, combined with postoperative radiotherapy and chemotherapy and targeted drug therapy, that survival time may be prolonged.

An accurate preoperative assessment of the tumor should be conducted to determine whether the patient should undergo primary tumor resection or whether there are unresectable distant metastases. In 215 patients prospective observational study, Maupoey Ibáñez et al. studied that for high-risk tumours (T3 $\geq 5 \mathrm{~mm}$ and T4), CTC showed an accuracy, sensitivity, and specificity of $82.7 \%, 86 \%$ and $80 \%$, respectively (19). Catalano et al. found that FDG PET/MRI compared with FDG PET/CT was superior for staging allowing accurate local and overall staging and restaging in a significant number of patients in colorectal cancer (20). Imaging examinations are necessary to confirm the size of the tumor and its relationship with adjacent organs. A CT scan of the chest, abdomen, and pelvis with maximum oral and contrast to evaluate the extent of the abdominal wall tumor is essential (21). CT and MRI examinations can help understand the tumor tissue density and blood supply. A three-dimensional reconstruction intuitively displays the location and adjacent relationships of the tumor, and provides important information about the formulation of the scope of surgical resection. Threedimensional visualization (3DV) technology can transform two-dimensional images into volume images and display the relationship between abdominal wall tumors and surrounding tissues or organs in an all-around, multi-angle, and transparent manner. Selected aspects of specific lesions, especially the invasion of abdominal wall tumors to adjacent tissues or organs, can be rotated to observe the distance in a three-dimensional plane at multiple angles, in order to assess whether the operation can proceed smoothly. 3DV, as a new technical method, plays an important role in preoperative evaluation and surgical planning, and has been used in liver and pancreatic surgery (22). In our study, all patients underwent preoperative three-dimensional reconstruction to accurately assess the condition of the tumor and its adjacent organs so as to avoid intraoperative risks (Figure 3).

According to our classification of abdominal wall defects (23), those caused by locally advanced CRC with abdominal wall invasion often belong to type II or type III. The main treatment plan is based on mesh reinforcement or bridge technology with component separation or myocutaneous flap technology. At present, the implant mesh materials primarily include synthetic non-degradable and biodegradable meshes. A synthetic non-degradable mesh can provide a permanent repair of the abdominal wall. However, it is not suitable for abdominal wall defects with possible infections. Biodegradable mesh could support neovascularization and host cell ingrowth, and the collagen matrix will be replaced by the body's own tissue, allowing for the possible reconstruction of abdominal wall defects with infection, especially for severely exposed patients.

The component separation technique (CST) is a method that releases the entire abdominal wall myofascial layer by separating a certain myofascial layer of the abdominal wall to achieve the purpose of closing a large abdominal wall defect. The CST mainly includes the anterior approach CST and the transversus abdominis release (TAR) technique of the posterior approach. The implementation of CST technology is relatively simple. One side of the CST can obtain up to $10 \mathrm{~cm}$ of myofascial tissue release in the mid-abdomen (24). TAR technology is primarily used to achieve release of the abdominal wall myofascial tissue through an incision in the transverse abdominis muscle, and can achieve $8-12 \mathrm{~cm}$ of myofascial release to reconstruct the abdominal wall defect (25).

For abdominal wall defects that do not have the appropriate conditions or are too large to be closed, bridging repair methods can also be considered (26). For type III abdominal wall defects with a full-thickness absent abdominal wall, autologous tissue transplantation is the first choice. Depending on the defect site, different pedicled flaps can be chosen, including the tensor fasciae latae (TFL), the rectus abdominis flap, the oblique muscle flap, the latissimus dorsi flap, and the rectus femoris muscle. These flaps can repair the myofascial layer and cover the skin at the same time. However, the free myocutaneous flap needs to be reconstructed and anastomosed using microsurgical techniques, and thus needs to be performed by a surgeon with special skills, which restricts the use of these applications. Since the transplanted tissue does not have sufficient mechanical strength, the incidence of postoperative hernia can be as high as $29 \%$ (24). Therefore, in our study, the repair methods for type III abdominal wall defects were mainly a combination of autologous tissue with a synthetic non-degradable or biodegradable mesh.

Based on the mesh, the CST or TAR, and flaps technology, we had enough confidence to complete the repair or reconstruction of a huge abdominal wall defect (23). However, an accurate and comprehensive preoperative assessment of patients with locally advanced CRC with abdominal wall invasion was required.

The prevalence of COVID-19 can affect preoperative 



$\mathrm{E}$

$\mathrm{F}$


Figure 3 Three-dimensional reconstruction process. (A) Cross section of two-dimensional plane; (B) cross section of three-dimensional plane; (C) bone reconstruction; (D) abdominal wall reconstruction; (E) top view; (F) simulation of abdominal wall defect after tumor resection.

screening for cancer and prevent patients from receiving timely treatment. In a study, Chen et al. screening for all 3 cancers declined sharply in March through May of 2020 compared with 2019, with the sharpest decline in April (breast, $-90.8 \%$; colorectal, $-79.3 \%$; prostate, $-63.4 \%$ ) in America (27). Public health efforts are needed to address the large cancer screening deficit associated with the COVID-19 pandemic, including increased use of screening modalities that do not require a procedure.

Due to the low incidence of locally advanced colorectal cancer with abdominal wall invasion, the sample size of this study was limited and few patients in the study were followed for a short time. The statistical results of this study have certain limitations.

\section{Conclusions}

The treatment of locally advanced CRC with abdominal wall invasion is quite complicated. A preoperative evaluation and preparation, multidisciplinary cooperation, and reasonable selection of surgical method are vital to ensure that patients receive effective treatment.

\section{Acknowledgments}

Funding: This work was supported by the National Natural Science Foundation of China (81970455), the Shanghai Science and Technology Committee (20Y11909100) and the Medical and Industrial Cross Research Fund of Shanghai Ninth Hospital (JYJC201914). The funders had no role in the study design, data collection and analysis, decision to publish, or preparation of the manuscript.

\section{Footnote}

Reporting Checklist: The authors have completed the STROBE reporting checklist. Available at http://dx.doi. org/10.21037/atm-21-2094 
Data Sharing Statement: Available at http://dx.doi. org/10.21037/atm-21-2094

Conflicts of Interest: All authors have completed the ICMJE uniform disclosure form (available at http://dx.doi. org/10.21037/atm-21-2094). The authors have no conflicts of interest to declare.

Ethical Statement: The authors are accountable for all aspects of the work in ensuring that questions related to the accuracy or integrity of any part of the work are appropriately investigated and resolved. This study was performed in accordance with the ethical standards of our institutional research committee and the principles of the Declaration of Helsinki (as revised in 2013). This study was approved by the ethics committee of the Ninth People's Hospital Affiliated to Shanghai Jiaotong University (approval number: 2016-128-177). Informed consents were obtained from patients.

Open Access Statement: This is an Open Access article distributed in accordance with the Creative Commons Attribution-NonCommercial-NoDerivs 4.0 International License (CC BY-NC-ND 4.0), which permits the noncommercial replication and distribution of the article with the strict proviso that no changes or edits are made and the original work is properly cited (including links to both the formal publication through the relevant DOI and the license). See: https://creativecommons.org/licenses/by-nc-nd/4.0/.

\section{References}

1. Arnold M, Sierra MS, Laversanne M, et al. Global patterns and trends in colorectal cancer incidence and mortality. Gut 2017;66:683-91.

2. Allemani C, Weir HK, Carreira H, et al. Global surveillance of cancer survival 1995-2009: analysis of individual data for 25,676,887 patients from 279 population-based registries in 67 countries (CONCORD-2). Lancet 2015;385:977-1010.

3. National Health Commission of the People's Republic of China. Chinese Protocol of Diagnosis and Treatment of Colorectal Cancer (2020 edition). Zhonghua Wai Ke Za Zhi 2020;58:561-85.

4. Biasco G, Derenzini E, Grazi G, et al. Treatment of hepatic metastases from colorectal cancer: many doubts, some certainties. Cancer Treat Rev 2006;32:214-28.

5. Mitry E, Guiu B, Cosconea S, et al. Epidemiology, management and prognosis of colorectal cancer with lung metastases: a 30-year population-based study. Gut 2010;59:1383-8.

6. Guend H, Patel S, Nash GM. Abdominal metastases from colorectal cancer: intraperitoneal therapy. J Gastrointest Oncol 2015;6:693-8.

7. van Gestel YR, de Hingh IH, van Herk-Sukel MP, et al. Patterns of metachronous metastases after curative treatment of colorectal cancer. Cancer Epidemiol 2014;38:448-54.

8. Reilly WT, Nelson H, Schroeder G, et al. Wound recurrence following conventional treatment of colorectal cancer. A rare but perhaps underestimated problem. Dis Colon Rectum 1996;39:200-7.

9. Ludmir EB, Palta M, Willett CG, et al. Total neoadjuvant therapy for rectal cancer: An emerging option. Cancer 2017;123:1497-506.

10. Sonbol MB, Mountjoy LJ, Firwana B, et al. The Role of Maintenance Strategies in Metastatic Colorectal Cancer: A Systematic Review and Network Meta-analysis of Randomized Clinical Trials. JAMA Oncol 2020;6:e194489.

11. Tomasello G, Petrelli F, Ghidini M, et al. FOLFOXIRI Plus Bevacizumab as Conversion Therapy for Patients With Initially Unresectable Metastatic Colorectal Cancer: A Systematic Review and Pooled Analysis. JAMA Oncol 2017;3:e170278.

12. Li A, Wu B, Cui L, et al. Successful en bloc resection of recurrent hepatocellular carcinoma directly invading the abdominal wall: a case report. J Med Case Rep 2015;9:19.

13. Takano M, Nishimura $Y$, Ishikawa $H$, et al. A Case of Long-Term Survival after Nine Surgeries for Recurrent Sigmoid Colon Cancer. Gan To Kagaku Ryoho 2015;42:2190-2.

14. Engstrand J, Kartalis N, Strömberg C, et al. The Impact of a Hepatobiliary Multidisciplinary Team Assessment in Patients with Colorectal Cancer Liver Metastases: A Population-Based Study. Oncologist 2017;22:1067-74.

15. Biller LH, Schrag D. Diagnosis and Treatment of Metastatic Colorectal Cancer: A Review. JAMA 2021;325:669-85.

16. Xu J, Fan J, Qin X, et al. Chinese guidelines for the diagnosis and comprehensive treatment of colorectal liver metastases (version 2018). J Cancer Res Clin Oncol 2019;145:725-36.

17. Tsilimigras DI, Brodt P, Clavien PA, et al. Liver metastases. Nat Rev Dis Primers 2021;7:27.

18. Yang ZF, Wu DQ, Wang JJ, et al. Short- and long-term outcomes following laparoscopic vs open surgery for 
pathological T4 colorectal cancer: 10 years of experience in a single center. World J Gastroenterol 2018;24:76-86.

19. Maupoey Ibáñez J, Pàmies Guilabert J, Frasson M, et al. Accuracy of CT colonography in the preoperative staging of colon cancer: a prospective study of 217 patients.

Colorectal Dis 2019;21:1151-63.

20. Catalano OA, Coutinho AM, Sahani DV, et al. Colorectal cancer staging: comparison of whole-body PET/CT and PET/MR. Abdom Radiol (NY) 2017;42:1141-51.

21. Esquivel J, Sticca R, Sugarbaker P, et al. Cytoreductive surgery and hyperthermic intraperitoneal chemotherapy in the management of peritoneal surface malignancies of colonic origin: a consensus statement. Society of Surgical Oncology. Ann Surg Oncol 2007;14:128-33.

22. Society Of Digital Medicine Chinese, Cancer Committee Of Chinese Medical Doctor Association Liver, Precision Medicine Committee Of Chinese Medical Doctor Association Clinical, et al. Clinical practice guidelines for precision diagnosis and treatment of complex liver tumor guided by three-dimensional visualization technology (version 2019). Nan Fang Yi Ke Da Xue Xue Bao 2020;40:297-307.

Cite this article as: Song Z, Yang D, Song H, Dong W, Wu J, Yang J, Gu Y. Surgical treatment strategy for locally advanced colorectal cancer with abdominal wall invasion. Ann Transl Med 2021;9(10):874. doi: 10.21037/atm-21-2094
23. Gu Y, Wang P, Li H, et al. Chinese expert consensus on adult ventral abdominal wall defect repair and reconstruction. Am J Surg 2020. [Epub ahead of print]. doi: 10.1016/j.amjsurg.2020.11.024.

24. de Vries Reilingh TS, Bodegom ME, et al. Autologous tissue repair of large abdominal wall defects. Br J Surg 2007;94:791-803.

25. Oprea VC, Rosian M, Mardale S, et al. Is transversus abdominis muscle release sustainable for the reconstruction of peritoneal volumes? A retrospective computed tomography study. International Journal of Abdominal Wall and Hernia Surgery 2020;3:25-33.

26. Patel KM, Nahabedian MY, Albino F, et al. The use of porcine acellular dermal matrix in a bridge technique for complex abdominal wall reconstruction: an outcome analysis. Am J Surg 2013;205:209-12.

27. Chen RC, Haynes K, Du S, et al. Association of Cancer Screening Deficit in the United States With the COVID-19 Pandemic. JAMA Oncol 2021. [Epub ahead of print]. doi: 10.1001/jamaoncol.2021.0884.

(English Language Editor: A. Kassem) 